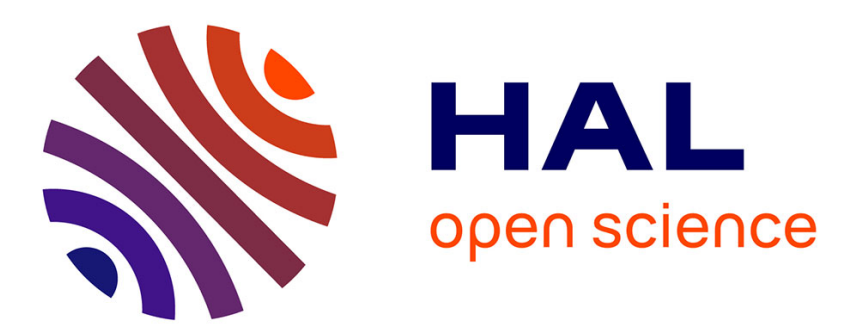

\title{
Biofilm research using calorimetry - a marriage made in heaven
}

Friederike Buchholz, Hauke Harms, Thomas Maskow

\section{To cite this version:}

Friederike Buchholz, Hauke Harms, Thomas Maskow. Biofilm research using calorimetry - a marriage made in heaven. Biotechnology Journal, 2010, 5 (12), pp.1339. 10.1002/biot.201000287 . hal00599453

\section{HAL Id: hal-00599453 \\ https://hal.science/hal-00599453}

Submitted on 10 Jun 2011

HAL is a multi-disciplinary open access archive for the deposit and dissemination of scientific research documents, whether they are published or not. The documents may come from teaching and research institutions in France or abroad, or from public or private research centers.
L'archive ouverte pluridisciplinaire HAL, est destinée au dépôt et à la diffusion de documents scientifiques de niveau recherche, publiés ou non, émanant des établissements d'enseignement et de recherche français ou étrangers, des laboratoires publics ou privés. 


\section{Biofilm research using calorimetry - a marriage made in} heaven

\begin{tabular}{|r|l|}
\hline Journal: & Biotechnology Journal \\
\hline Manuscript ID: & biot.201000287.R1 \\
\hline Wiley - Manuscript type: & Review \\
\hline Date Submitted by the & $29-$ Oct-2010 \\
\hline Complete List of Authors: & $\begin{array}{l}\text { Buchholz, Friederike; UFZ-Helmholtz-Centre for Environmental } \\
\text { Research, Environmental Microbiology } \\
\text { Harms, Hauke; UFZ-Helmholtz-Centre for Environmental Research, } \\
\text { Environmental Microbiology } \\
\text { Maskow, Thomas; UFZ - Helmholtz-Centre for Environmental } \\
\text { Research, Environmental Microbiology }\end{array}$ \\
\hline Primary Keywords: & Biosensors \\
\hline Secondary Keywords: & Thermodynamics \\
\hline Keywords: & Monitoring, Calorimetry, Disinfectants \\
\hline
\end{tabular}

\section{SCHOLARONE $^{\text {TH }}$ Manuscripts}




\section{Biofilm research using calorimetry - a marriage made in heaven?}

Friederike Buchholz; Hauke Harms; Thomas Maskow

UFZ - Helmholtz Centre for Environmental Research, Dept. Environmental Microbiology, Leipzig, Germany

Key words: Biosensors, Thermodynamics, Monitoring, Calorimetry, Disinfectants

*Corresponding author:

PD Dr. Thomas Maskow

UFZ - Helmholtz Centre for Environmental Research, Dept. Environmental Microbiology, Permoserstr. 15,

04318 Leipzig,

Germany

Fax: +49-341-235 1351; Tel.: +49-341-235 1328

E-mail address: Thomas.maskow@ufz.de 


\begin{abstract}
Bacteria growing surface-associated, so-called biofilms, play an important role in technical processes like waste water treatment, bioremediation, or bioprocessing. On the contrary, problems arise when biofilm growth results in e.g. clogged pipes, microbially influenced corrosion or pathogenic contamination, undesired processes that may cause huge financial losses. For observation purposes and to develop efficient control strategies, real time monitoring tools for biofilms are required. Among the large variety of tools used in biofilm research, calorimetry is rarely applied, even though many characteristics qualify it for biofilm investigation and monitoring. Calorimetric measurements are non-invasive and nondestructive and applicable to nearly any kind of samples (including heterogeneous or turbid solutions) without the need of special sample preparation. Online and real time data acquisition reduces the labour and facilitates high throughput measurements.

The following article is meant to introduce and promote calorimetry as a future tool in biofilm research. It attempts an assessment of common, existent monitoring tools and specifically addresses the potential of calorimetry in this field.
\end{abstract}

\title{
1) Urgent demand in biofilm monitoring tools
}

The vast majority of bacteria in natural environments does not occur in well aerated nutrient rich suspensions, as many microbiological laboratory studies appear to suggest, but thrives on surfaces or phase-interfaces. The interaction between bacteria and surfaces had been described in the literature for many years [1-3] but it was in the 1970s that the term "biofilm" for bacteria, living attached to surfaces or interfaces and embedded in a polymeric matrix, was introduced. Not least thanks to this concise and handy term, a blooming research field with ever increasing numbers of publications has developed since then. 
Biofilm bacteria exhibit physiological characteristics differing from those of their freefloating counterparts. The altered physiological behaviour leads to a better stability against extreme and fluctuating environmental conditions such as $\mathrm{pH}$, temperature, salinity, water activity, bioavailability of both nutrients and harmful chemical agents like disinfectants or antibiotics. In some industrial processes, biofilm cultures are preferred as they provide higher process stability $[4,5]$. On the downside, they are very difficult to eradicate wherever their occurrence is undesired. The enhanced resistance can be attributed to the protective function of the extracellular polymeric substances and to other physiological adaptations like slow growth, the production of exoenzymes, or the activity of multidrug-efflux-pumps. Besides, the occurrence of persister cells is also discussed. Persister cells are non-growing, spore-like cells that are nearly impervious to antimicrobial effects. Biofilm persistence may thus rely on the random conversion of common cells to persister cells. For detailed review of the different protective mechanisms the reader is referred to $[6,7]$.

The unusual behavior of biofilm bacteria represents a challenge for the development of control strategies. Specific investigation methods are needed for biofilms, which have to account for the particular growth mode.

Since long, it is known that biofilm-bacteria, due to undesired growth on surfaces (e.g. heat exchangers, ship hulls, pipes for drinking- and process-water, medical implants), are involved in many detrimental processes like corrosion, energy losses or medical complications, thereby leading to huge economical losses. This gave rise to an intensive search for biofilm monitoring and control methods. The interest in such methods was further enhanced by the application of surface-associated microorganisms in technical systems (e.g., in biotechnological or bioremediation processes), municipal wastewater treatment, biodegradation of organic waste, bioprocessing and food fermentation procedures. Further beneficial and essential aspects of biofilms motivated the desire to better understand these bacteria. Additional drivers of the latter research were their involvement in the natural 
purification of water [8], their functions as physiological flora on higher animals, including humans where they provide protection against pathogens $[9,10]$ and their roles in the digestive system of animals where they are responsible for vitamin synthesis or the degradation of specific substances such as lignin $[11,12]$

Monitoring tools are required under all circumstances where biofilms either cause problems or are beneficial. They are needed for the improvement of biofilm-based bioprocesses as well as for the evaluation of the success of countermeasures against biofilms. Furthermore, techniques are needed to achieve a better scientific understanding of the complex biofilm communities.

\section{2) Existing biofilm monitoring tools - different kinds and levels of information}

Different situations require different kinds and levels of biofilm control. For example in industrial systems, biofilm growth cannot be completely prevented and therefore has to be kept in a range where the system is not negatively influenced. In contrast, complete sterility is required for most medical applications [13].

The available monitoring tools can be classified according to the attained level of information $[13,14]$. The following paragraphs will give a short overview on available methods, more detailed information is given in $[13,14]$. It has to be noticed that there are advantages for every method under certain conditions, although under most practical circumstances broadly applicable methods are desired.

The simplest type of monitoring devices detects mass deposition on surfaces, regardless if it is biotic or abiotic in nature, alive or dead. Common examples are optical methods $[15,16]$ which are used to detect changes of biofilm thickness on a surface according to dispersed or reflected light density. These methods can be influenced by the concentration of particulate matter, the colour of the biofilm or the chemical composition of the water. Another possibility is the direct colorimetric measurement of biofilm accumulation on surfaces [17]. This method 
infers cell density from the surface discoloration arising during biofilm growth. It works nondestructively and provides immediate results, but a calibration to the surface is necessary and the measurement is influenced by the types of bacteria and chemicals used. Interferences with coloured abiotic materials can also not be excluded. This makes the investigation of unknown samples difficult although it might be useful for experiments under well known conditions. Many more methods of this type are described in [13]. Even though the information provided by these simple monitoring devices is rather low, so that confirmation by other methods is required, they might be very useful for early indication of biofouling in technical settings.

Somewhat more sophisticated techniques can distinguish between biotic and abiotic depositions. Commonly used methods of this kind are the (Infrared) IR- or (Fourier Transform Infrared - Attenuated Total Reflectance) FTIR-ATR spectroscopy of amid bands and microscopic techniques. IR- or FTIR-ATR of amid bands does not require sample preparation but can only be applied in systems free or poor of other biological molecules. Moreover, only smooth surfaces are suited for this kind of analysis. Other methods of similar information content, i.e. direct light-microscopy of biofilms without the application of specific dyes, can sometimes hardly distinguish between microorganisms and attached abiotic material. Biofilm staining with, for instance, crystal violet or safranin followed by washing, color extraction and absorption measurements of the extract, give a rough estimation of biofilm mass and differentiate between biotic and abiotic material, but they do not give information about viability or activity [18].

The deepest insight into biofilms is offered by techniques that provide detailed information about the chemical composition or directly address biofilm microorganisms like e.g. FTIRATR of whole spectra in special flow cells or direct biofilm visualization by confocal-laserscanning-microscopy (CLSM) or epifluorescence-microscopy. Modern microscopic techniques rely on the fluorescence of added dyes or probes. These dyes may interact with the bacterial metabolism. Furthermore, the exact staining behavior is uncertain when they are 
applied to unknown environmental samples. Moreover, in spite of their high costs, many dyes are toxic and require environmentally friendly disposal. The invasive nature of secondary staining-procedures excludes the application of modern microscopic techniques for real time monitoring in most cases, although the gained snapshot information may be of high diagnostic value.

Microelectrodes are also commonly used in biofilm research. However, for monitoring purposes they are not particularly suited. The insertion of electrodes is destructive, even though tips with a diameter of a few $\mu \mathrm{m}$ are nowadays available. The electrodes are analyteselective and available for instance for different terminal electron acceptors (e.g. oxygen, nitrate, and nitrous oxide), electron donors (e.g. sulphide, glucose), nutrients (e.g. ammonium), toxicants (e.g. chlorine) and environmental conditions (e.g. pH). In any case, careful calibration is necessary. The provided information only refers to the area where the electrode is inserted and the origin of the detected substances, biotic or abiotic, is not distinguished. Specially in environmental biofilm samples of unknown composition, signal interpretation is difficult. Furthermore, working with microelectrodes implicates dealing with strong responses to interferences and signal drifts.

Nuclear magnetic resonance (NMR) methods have been used for non-invasive imaging of biofilms in porous media and to obtain information about intermediate and metabolite profiles [19-21]. For industrial and field applications is NMR too expensive and not flexible enough to be integrated into technical and natural systems of interest.

Calorimetry is directly addressing the activity of biofilm microorganisms by measuring their metabolic heat. Despite the advantageous characteristics of calorimetry, which qualifies it as a promising monitoring tool for biofilm research, there are only few applications published. These examples and their information gain in comparison to conventional methods will be discussed in detail in chapter 3. 
The most frequently applied technique in biofilm investigations is the determination of colony forming units (CFU). Here biofilms are removed by scraping, vortexing or sonication, resuspension, and recultivation on agar-plates followed by counting of growing colonies [22]. The procedure is slow and error-prone due to the possibility of incomplete resuspension before plating or carryover of substances from the liquid medium [23]. Furthermore, biofilm cells exhibit a specific physiological state which may result in a situation that most of them do not grow after plating on agar (W. Costerton, personal communication). In comparison with cultivation independent methods, CFU typically underestimate cell counts by at least one order of magnitude [24]. Therefore, all results based on determination of CFU derived from resuspended biofilms are highly doubtful. Generally, cultivation steps require around $24 \mathrm{~h}$ until results become available. This disqualifies cultivation-based methods wherever quick responses, e.g. in medical applications, are needed.

The ideal biofilm monitoring method should work in aqueous systems and function without sample removal. Secondary procedures like recultivation or staining should be dispensable. Furthermore, real time and automated online data acquisition should be possible. It should also be possible to investigate various sample types on various surfaces under various conditions (environmental/technical samples). Finally, integration into technical systems should be feasible.

\section{3) Biofilm monitoring via heat: calorimetry}

\section{1) Basic concepts of calorimetry}

Calorimetry is defined as any type of measurement of heat, irrespective if it is evolved (exothermic) or required (endothermic), by any physical, chemical or even biological process. Due to the fact that all chemical reactions, including those of the metabolic network, are accompanied by release or consumption of heat, calorimetry provides real-time information 
about the metabolically active cells. It embraces both, the kinetics as well as stoichiometric information of the growth reactions. The obtained data have to be interpreted with caution, because the heat signal also comprises chemical side reactions or physical processes occurring in the sample, like e.g. phase transitions. However, if all chemical and physical processes are known and taken into account, the data interpretation is easy [25]. Calorimetric measurement is non-invasive and non-destructive. The most important feature is that the measured heat reflects the ongoing processes in real time. The online data acquisition and the easy and fast operating steps qualify calorimetry as a perfect tool for biofilm analysis and monitoring. Furthermore the obtained signals have a quantitative meaning and do not require any calibration, unlike many other methods. Additionally, thermodynamic calculations based on Hess` law can prove expected or reveal unexpected reactions or side effects. Another advantage is that calorimetric experiments can be done in a broad range of measuring conditions regarding for instance $\mathrm{pH}$-value, temperature, electron-acceptor or sample character. Calorimetry has already been used successfully for the monitoring of suspended microbial cells for many decades [26, 27].

Surprisingly, calorimetry was until now seldom applied to biofilm research. The reasons might lie in the difficulty of analysing biofilms using conventional and commercially available calorimeters. However, newly developed calorimeters promise successful and easy application to biofilm analysis and monitoring. However, pioneering work was done with conventional calorimeters [28-31].

For biofilm measurements, mostly flow systems were chosen [28, 30, 31], even though also static experiments in ampoules have been conducted [32, 33]. Modifications of fermentation calorimeters could have been another option for calorimetry in biofilm research. However, except for experiments with sludge $[34,35]$ no reports about biofilm research in fermentation calorimeters can be found in the literature. Recently developed chip-calorimeters, equipped with exchangeable flow-chambers, allow a higher sample throughput than conventional 
microcalorimeters. The basic measurement principle of micro- or chip-calorimeters is that the heat produced in a sample flows through thermopiles (consisting of coupled Peltier-elements) towards a heat sink with a well defined temperature (thermostat). The Peltier elements act as thermoelectric generators and convert, according to the Seebeck-effect, the temperature difference between the sample and a reference point into a voltage signal which is proportional to the heat. Figure 1 shows a scheme and a photograph of the measurement position of a conventional microcalorimeter (A) and a chip-calorimeter (B).

\section{Here Figure 1}

In case (A) the sample is located either in a measurement vessel (mainly static conditions) or in a flow channel in the wall of the measurement cylinder. In case (B) it is located in a flowcell. The sensitivity of the methods differs, e.g. commercially available microcalorimeter have a sensitivity of about $0.05 \mathrm{~mW} / \mathrm{L}$, the chip-calorimeter recently developed by Lerchner et al. 2008 [36] has a sensitivity of about $3 \mathrm{~mW} / \mathrm{L}$.

The main deficiency of established microcalorimeters regarding biofilm research is that either the measurements have to be done in a static manner or in a flow channel. Static measurements do not reflect the real situation of the biofilm because the heat produced by planktonic cells adds to the heat produced by the biofilm cells. Flow-through measurements are time consuming because the biofilm has to develop inside the calorimeter, which can require days to weeks to reach a stable state. During this time a calorimetric vessel is blocked for the measurement of a single biofilm. Any solution overcoming these deficiencies would facilitate the application of conventional microcalorimeters in biofilm research.

As shown in recent publications [36, 37] chip-calorimeters overcome some deficiencies of conventional microcalorimeters and are thus developing into a very valuable tool for biofilm investigations. They employ flow cells, which are general tools in biofilm research. 
Exchangeable flow-cells allow the separation of biofilm cultivation and calorimetric measurement, thus permitting much higher throughput. A weakness is that the biofilm in the flow cell can not continuously be flushed with nutrient solution. A stop flow regime is required to obtain a reference state. Low heat production due to oxygen consumption is considered as reference state. Details are described in Lerchner et al. 2008 [36].

\section{2) History of biocalorimetry}

\section{General applications of calorimetry in microbiology}

Calorimetry has been used as an analytical tool in microbiology for a long time. One of the earliest calorimetric experiments involving microorganisms took place in 1856 when Augustine Pierre Dubrunfaut determined the heat of alcoholic fermentation of Saccharomyces cerevisiae in an oak vat [38]. The heat production due to other fermentation processes (e.g the souring of milk) was recorded in 1911 [39]. In 1912, Meyerhoff combined measurements of heat, consumed $\mathrm{O}_{2}$ and evolved $\mathrm{CO}_{2}$ and used the ratio of the produced heat to the consumed oxygen to distinguish different phases of microbial growth [40]. Today this ratio is referred to as the oxycaloric quotient [41]. Bayne-Jones et al. in 1929 [42] correlated the heat produced by bacterial growth and cell counts and found that changes in heat production are related to changes in the microbial growth rate. Decades later, calorimetry was used for more sophisticated quantitative investigations of microbial growth. From the relations of calorimetrically measured enthalpy changes of microbial growth, the calculated Gibb's energies and the observed growth yields and rates, thermodynamic methods to predict maximum possible yields, maintenance coefficients, threshold concentrations and maximum growth rates were derived [43]. Recently, very successful applications of calorimetry in the medical field (e.g. analysis of bacterial infections of blood, body fluids or implants [44-46]) have been reported. Further application examples are reviewed in [27, 47]. 
The amount of heat produced by bacterial cells varies, depending on the prevailing type of metabolism. Reported values for heat production per cell in suspensions are in the range of $0.9 \mathrm{pW} /$ cell (methanogenesis) [48] and approx. $7.8 \mathrm{pW} /$ cell (E. coli aerobically growing in complex medium) [49]. For surface-associated cells only very few data are available. Lerchner et al. reported $0.13 \mathrm{pW}$ per cell [36], whereas from the results of von Rège et al. [29] a heat output of $0.42 \mathrm{pW}$ per cell can be calculated. Supposedly, the heat output per biofilm cell varies as much as it does for suspended cells, maybe even more because there are different maturation stages of biofilms. Therefore, the difference between the reported values is not surprising.

\section{Biofilm related applications of calorimetry}

The first calorimetric approach involving surface attached microbial cultures was to our knowledge done by Weppen et al. in 1991 [31]. They cultivated microorganisms on the surface of glass beads and used these microbial cultures in a microcalorimeter for the onlineestimation of the biological oxygen demand of aqueous samples. Short time later Wentzien et al. in 1994 [30] investigated thiosulfate and tetrathionate degradation by Thiobacillus species in a flow through-microcalorimeter. They were able to detect metabolic changes due to adherence on the surface of the measurement cylinder as well as the contribution of biofilm cells to thiosulfate and tetrathionate degradation relative to suspended cells.

\section{Here Fig. 2}

Both curves in Fig. 2 show the heat output of T. intermedius growing on thiosulfate. The difference is due to the different treatments of the flow lines in the measurement cylinders. In case of the graph marked with triangles (I) bacterial suspension was continuously pumped through the flow line. Thus, the heat signal is the sum of the metabolic heat of planktonic and 
surface associated growing bacteria. In case of the graph with rectangles (II) the flow line was periodically rinsed with disinfection solution to remove surface associated bacteria. Because of the disinfection steps, the measurement was interrupted and the graph is constructed from separate data points. Graph I shows two maxima and the results of the parallel metabolite analysis suggest that it is rather not reflecting two steps of a degradation process. The heat could be released due to physical interactions between the bacterial surface and the surface of the flow-line, or due to a stimulation of the metabolism, induced by adherence. Since the processes are very complicated and overlapping, the reasons for the increase in heat production can only be speculated about. However, the difference in the heat output between graph I and II clearly indicates that the bacteria adhere to the surface. In the same way the impact of medium components or surface material on adhesion and biofilm development can be evaluated. Bacterial adhesion to surfaces as well as coaggregation were also detected microcalorimetrically by Hauser-Gerspach et al. [33] and Postollec et al. [50] who investigated adhesion and aggregation behaviour of dental streptococci. Morgan et al. [51] suggested a microcalorimeter with a modified acrylic ampoule as a model system to evaluate the efficiency of adhesion to different surfaces and of anti-biofilm treatments.

In a study of von Rège et al. biocide efficiency was evaluated via a microcalorimetric approach [29]. They performed ampoule experiments with biofilms, precultivated on steel coupons (static conditions), and flow-through experiments (dynamic conditions). Different biocides were tested and decrease in activity was compared to cell counts. These authors showed that microcalorimetry has considerable advantages compared to common evaluation methods of bactericidal effects. They suggested that microcalorimetry could be a useful control tool for biofilm growth in technical systems or for the development and testing of new biocides.

Most calorimetric biofilm investigations were performed with mixed cultures. However, this complicates the correlation of observed heat signals and metabolic events. This could be a 
reason why before 2007 it was, to our knowledge, never attempted to use the additional information of an energy-balance for biofilm investigations. In 2007 Peitzsch et al. [28] performed the first attempt to calculate an energy balance of a single species biofilm of E. coli in a defined medium with a single carbon source (i.e. glycerol). Measurements were carried out in a TAM-microcalorimeter. An energy consumption rate was calculated from the carbonsubstrate consumption and correlated with the heat production rate. For a younger biofilm $(<150 \mathrm{~h})$ similar patterns for energy consumption rate and heat production rate were reported. For more mature biofilms the energy consumption rate decreased whereas the heat production rate remained constant. Thus, the efficiency of energy usage increased. Furthermore, they reported for the first time about the calorimetric detection of detachment events. The graph in Fig. 3 (based on the data of Peitzsch et al. [28]) shows the heat production rate of the biofilm combined with the carbon distribution. In the early phase (until $100 \mathrm{~h}$ ) the heat signal shows only a few small peaks, whereas after $100 \mathrm{~h}$ many sharp increases in heat production rate can be observed. These peaks are accompanied by an increase in the ratio between the measured TOC (Total Organic Carbon) in the outflow and the calculated and expected TOC (carbonsource glycerol and the known side product acetate). The authors suggested the detachment of biofilm flocs as an explanation. This can explain both, the increased amount of unknown TOC as well as the increased heat because of increased metabolic activity in the biofilm flocs due to better oxygen availability.

\section{Here Fig. 3}

Additionally, Peitzsch et al. [28] monitored the toxic influence of 2,4-dinitrophenol (DNP). The metabolic heat signals, in response to different 2,4-DNP concentrations and to short-term and continuous intoxication were clearly distinguishable. 
In 2006, Maskow et al. applied for the first time a new calorimetric technique (i.e. miniaturized- or chip-calorimetry) for biofilm investigations. Advantages of this handy instrument were higher flexibility and lower costs compared to conventional calorimeters. They monitored the growth of an E. coli biofilm using the chip-calorimeter and a conventional calorimeter and showed the applicability of chip-calorimetry to qualitative biofilm monitoring [47]. In 2008, the growth of a Pseudomonas putida biofilm was quantitatively monitored using a recently developed chip-calorimeter [36]. The detected heat signal was correlated to CLSM cell counts and oxygen consumption, indicating the quantitative potential of the method. To verify the calorimetric signals, the biofilm responses to carbon shortage and varied nutrition strategies were investigated.

With the same technique, bactericidal and bacteriostatic treatments of biofilms were explored [37]. Figure 4 shows the heat response of the Pseudomonas putida biofilm towards the bacteriostatic antibiotic tetracycline, the bactericidal antibiotic kanamycin and the biocide formaldehyde. After the addition of the antibiotics to the substrate solution the heat signal decreases, in case of tetracycline in a mainly linear way, in case of kanamycin in a slightly biphasic way. The different heat responses reflect the different killing mechanisms of the antibiotics. The bacteriostatic tetracycline kills only growing cells, and especially the slow growing biofilm bacteria are only poorly affected by the antibiotic. Kanamycin actively kills bacteria but it is reported to be influenced by extracellular polymeric substances of biofilms. Here the decrease of the signal intensity starts later but it is faster than in case of tetracycline. To demonstrate the fast response of the calorimetric method, a biofilm was treated with the biocide formaldehyde. The immediate inactivation of the biofilm bacteria was reflected in the calorimetric signal. Details are given in [37].

\section{Here Fig. 4}


The study clearly indicates the suitability of calorimetry for the monitoring of disinfection processes or antibiotic treatments. Further physiological responses were revealed by other features of the response curve, like for example the increased signal intensity right after the addition of the antibiotics. This probably reflected the increased energy demand due to activation of cellular resistance mechanisms.

All examples presented so far used biofilms which were cultivated under lab conditions, but calorimetry is also applicable to the investigation of environmental biofilms. One possibility is the exposure of coupons or other surfaces in natural habitats, which can be collected for measurements after microorganisms have settled there. Additionally, because calorimetry does not require a special sample type (liquid-solid, solid etc), samples can be taken with their natural substratum, transferred in the measurement chamber and measured. Soil particles represent the largest surface for bacterial settlement and biofilm growth in nature. Thus, it is not surprising that soil scientists take already advantage of the broad range of sample types which can be investigated in a calorimeter. Microbial activity in soil is regarded as a measure for fertility since microbes are the key players in biogeochemical cycles [52]. The activity of microorganisms is mainly influenced by soil organic carbon which contributes to $20 \%$ to the increase of atmospheric $\mathrm{CO}_{2}$ [53] thus linking the loss of soil fertility to global warming. Therefore, it is increasingly important to understand the soil carbon cycle and the impact of anthropogenic influence $[32,54]$. Calorimetry offers a fast and simple possibility for the assessment of the fate of soil organic carbon. The limiting factor is the number of active bacteria, that differs depending on the ongoing metabolic process and the used instrument. In most studies, soil samples are amended with glucose to promote bacterial growth. According to Kinura et al. the heat production in a glucose amended soil sample is about $6.7 \mathrm{pW}$ per cell, [55]. Assuming a thermal detection limit of $100 \mathrm{nW}$ (achievable with modern nanocalorimeters) and a calorimetric vessel load of $1 \mathrm{~g}$ (Elizabeth Sigstad, personal communication), a detection limit of around 15000 active cells per $\mathrm{g}$ is estimated. This 
qualifies calorimetry for the quantification of soils even from extreme habitats, which are only sparsely populated with biofilm bacteria.

Figure 5 is based on the data of Barros et al. [32] where the heat flow from different soil samples is compared. The soil samples were taken from different sites in the Amazonian region, varying in the mode of land use. The soil samples differ strongly in their composition, specially regarding the organic matter content, leading to different microbial communities. In response to the addition of glucose all soil samples showed different patterns of heat production rates. In combination with the biomass yield, the efficiency of carbon and energy conversion was determined. It varied strongly (37 - 84\%) depending on the mode of land use.

\section{Here Fig. 5}

As assumed, calorimetry offers a fast and easy method to evaluate microbial activity in soils. Factors influencing the microbial activity, as for instance soil humidity, soil chemical composition and the presence of fertilizers, pesticides or contaminants, can be easily investigated in a nearly undisturbed sample [52, 56]. For more details see Barros et al. [57]. Usually, ammonia besides of glucose is added to induce the metabolism and thus heat flow. Considering that not all microorganisms are able to use glucose as substrate, e.g. many anaerobic bacteria, this approach might provide only limited information. Combined measurements of heat production and $\mathrm{CO}_{2}$-evolution can provide deeper insights (e.g. indicate the efficiency of the carbon substrate utilization for growth) [58]. The oxycaloric quotient for all aerobic heterotrophic growth processes is about $-455 \pm 15 \mathrm{~kJ}^{\mathrm{mol}-\mathrm{O}_{2}{ }^{-1}}$ [59]. Processes such as mixed respiratory-fermentative metabolic reactions or enhanced futile substrate cycling and uncoupling of oxidative phosphorylation result in a higher value of the oxycaloric quotient than the theoretical value for heterotrophic growth. 
Experiments with suspended cells have shown that it is possible to derive the kinetics of microbial degradation of contaminants from calorimetric data $[60,61]$ and that this could be applied in soil investigations to estimate the bio-remediation potential. Advantageous in this approach is the fact that the kinetics derived from calorimetric experiments reflects the metabolism, whereas kinetics derived from chemical analysis (e.g. HPLC or GC) reflects only the contaminant-removal from the aqueous soil phase. Chemical analysis includes also the disappearance due to sorption to soil matter. In most cases sorption processes do not affect significantly the heat signal, since the heat of sorption is many times smaller than the dissipated heat of biodegradation, therefore calorimetry is not biased by sorption processes. In comparison to chemical analysis calorimetry provides additional information about the fate of the contaminant (i.e. the bioconversion to incomplete oxidized substances).

\section{3) Potential application fields of calorimetric biofilm monitoring}

\section{Calorimetry as monitoring tool for disinfection processes or antibiotic susceptibility}

Biofilm-directed antibiotic susceptibility tests gained much attention, when it became clear that the different physiological characteristics of biofilm bacteria result in dramatic increases (1000 fold and more) in resistance towards antibiotics. For long time, susceptibility tests had been performed with suspended cells. Suddenly, new methods were required, which considered the mode of growth. When investigating biofilms, disruption, resuspension and recultivation should be avoided for reasons mentioned above. Johnston et al. compared the amount of surviving cells determined by the resuspension method and by the non invasive impedance method [62]. The amount of surviving cells was much higher than indicated by the recultivation experiments. This shows the importance of the integrity of the biofilms during the evaluation of disinfection treatment efficiency and quantification of surviving cells. Microcalorimetry has been applied successfully to determine MICs (minimal inhibitory concentrations) $[63,64]$. In this study the effect of various antibiotics towards $E$. coli and $S$. 
aureus strains was investigated calorimetrically and the results were compared to the standard method based on turbidity measurements. It clearly indicated that MIC determination via the heat signal works and even subinhibitory effects were revealed. First investigations concerning antibiotic influence on biofilm bacteria were done using chip-calorimetry (Fig. 4). These results clearly qualify this technique for the monitoring of antibiotic or disinfection treatments. Further research especially aiming at high throughput measurements is intended to be conducted in the near future.

\section{Calorimetry for the evaluation of biofilm-growth on surfaces}

For the attachment of bacteria, the initial step of biofilm growth, the character of the surface is very important.[65] Currently, much research is performed to develop materials which prevent bacterial attachment and biofilm growth [66]. Again, fast and easy methods are required for evaluating the efficiency of the new antimicrobial surface coatings or modifications. Regardless of its shortcomings, CFU determination is very often the method of choice. An attempt has been made for instance by Andersen and Co-workers who developed a flow-chamber test in which biofilm formation is determined microscopically using the well established crystal-violet staining [67]. In this approach even a flow regime was kept, which means a substantial progress compared to the often used microtiter-plate assays. However, microscopic approaches using special dyes or fluorescence-tagged probes (Fluorescence InSitu Hybridization - FISH) [68] can characterize biomass on surface only with limitations stated before. A replacement of these techniques would be desirable. The non-invasive and non-destructive character of calorimetry, as well as the smaller effort needed to perform the measurements, qualifies calorimetry as a tool for the evaluation of biofilm growth on surfaces. A recent publication applied calorimetry to investigate biofilm growth on different materials for bone transplantations [69] and further applications of this kind are to be expected. 


\section{4) The future of calorimetry in biofilm research}

For a broader application of calorimetry in biofilm research some technical and scientific challenges have to be overcome. There are several reasons, why this technique is not yet established in biofilm research, e.g. the high costs and the low throughput of the commercially available instruments, the limited flexibility and most likely the lack of expertise required handling and interpreting thermodynamic data.

Steps to improve the situation have been achieved with the development of chip-calorimeters. These instruments are cheaper than the former commercially available systems. They allow the separation of biofilm cultivation and measurement and increase the possible throughput. A development towards real high-throughput measurements has been made by Torres et al in 2004 [70]. They presented a low cost nano-calorimeter which can detect enthalpies of binding, enzymatic turnover, and other chemical reactions in arrays of 96 positions. Sample volume and measurement time are reduced by three orders of magnitude compared to a conventional calorimeter. They suggested further applications in the field of proteomics or drug discovery. The first commercially available high-throughput-calorimetric system was a device by the Belgian company Vivactis. This calorimeter variation performs measurements in microplate format (96-well). It works completely automated with robotic sample-handling and computerized data acquisition and data handling. The main problems of such multichannel chip-calorimeters are cross-talk effects between the channels and the large influence of water evaporation due to the open design of these arrays. For further information on enthalpy arrays and high throughput developments in calorimetry the reader may consult [71]. One approach of multichannel chip-calorimeters using whole eukaryotic cells was published by Verhaegen et al. in 2000 [72]. To transfer these developments to high throughput biofilm research, these methods have to be adapted carefully. 
A scientific challenge is the use of the thermodynamic heat data in combination with other methods to calculate energy balances for biofilm bacteria. Furthermore, most investigations have been done with lab cultures where the involved bacteria are known. The exposure of flow cells or designed substratum to the environment followed by calorimetric measurements would prove the applicability for environmental and ecological studies.

Recent developments in the field of photocalorimetry could even allow the investigations of phototrophic biofilms [73].

In summary, with the progress in modern calorimetry design, characterized by miniaturization, ultra sensitive measurement and high throughput ability, a promising biofilm monitoring tool entered the scientific scene. This measurement principle provides real time information about metabolically active cells. It is able to measure any type of sample and is flexible enough to be integrated into technical or natural systems of interest. 


\section{References}

[1] Zobell, C. E., The Effect of Solid Surfaces upon Bacterial Activity. J. Bacteriol. 1943, 46, $39-56$.

[2] Gristina, A. G., Costerton, J. W., Bacterial adherence and the glycocalyx and their role in musculoskeletal infection. Orthop Clin North Am 1984, 15, 517-535.

[3] Costerton, J. W., Cheng, K. J., Geesey, G. G., Ladd, T. I., et al., Bacterial biofilms in nature and disease. Annu Rev Microbiol 1987, 41, 435-464.

[4] Qureshi, N., Annous, B., Ezeji, T., Karcher, P., Maddox, I., Biofilm reactors for industrial bioconversion processes: employing potential of enhanced reaction rates. Microbial Cell Factories 2005, 4, 24-45.

[5] Gross, R., Hauer, B., Otto, K., Schmid, A., Microbial biofilms: new catalysts for maximizing productivity of long-term biotransformations. Biotechnol Bioeng 2007, 98, 1123 1134.

[6] Mah, T.-F. C., O'Toole, G. A., Mechanisms of biofilm resistance to antimicrobial agents. Trends in Microbiology 2001, 9, 34-39.

[7] Chambless, J. D., Hunt, S. M., Stewart, P. S., A three-dimensional computer model of four hypothetical mechanisms protecting biofilms from antimicrobials. Appl Environ Microbiol 2006, 72, 2005-2013.

[8] Sabater, S., Guasch, H., Romaní, A., Muñoz, I., The effect of biological factors on the efficiency of river biofilms in improving water quality. Hydrobiologia 2002, 469, 149-156.

[9] Hadaway, L. C., Skin Flora and Infection. Journal of Infusion Nursing 2003, 26, 44-48.

[10] Probert, H. M., Gibson, G. R., Bacterial biofilms in the human gastrointestinal tract. Curr Issues Intest Microbiol 2002, 3, 23-27.

[11] Breznak, J. A., Brune, A., Role of Microorganisms in the Digestion of Lignocellulose by Termites. Annual Review of Entomology 1994, 39, 453-487. 
[12] Brune, A., Miambi, E., Breznak, J. A., Roles of oxygen and the intestinal microflora in the metabolism of lignin-derived phenylpropanoids and other monoaromatic compounds by termites. Appl Environ Microbiol 1995, 61, 2688-2695.

[13] Flemming, H. C., Role and levels of real-time monitoring for successful anti-fouling strategies - an overview. Water Sci Technol 2003, 47, 1-8.

[14] Lewandowski, Z., Beyenal, H., Biofilm monitoring: a perfect solution in search of a problem. Water Sci Technol 2003, 47, 9-18.

[15] Bakke, R., Kommedal, R., Kalvenes, S., Quantification of biofilm accumulation by an optical approach. J Microbiol Methods 2001, 44, 13-26.

[16] Tamachkiarow, L., Flemming, H. C., On-line monitoring of biofilm formation in a brewery water pipeline system with a fibre optical device. Water Sci Technol 2003, 47, 19-24. [17] Pitts, B., Hamilton, M. A., McFeters, G. A., Stewart, P. S., et al., Color measurement as a means of quantifying surface biofouling. J Microbiol Methods 1998, 34, 143-149.

[18] Polonio, R. E., Mermel, L. A., Paquette, G. E., Sperry, J. F., Eradication of biofilmforming Staphylococcus epidermidis (RP62A) by a combination of sodium salicylate and vancomycin. Antimicrob Agents Chemother 2001, 45, 3262-3266.

[19] Gjersing, E. L., Herberg, J. L., Horn, J., Schaldach, C. M., Robert, S. M., NMR Metabolomics of Planktonic and Biofilm Modes of Growth in Pseudomonas aeruginosa. Analytical Chemistry 2007, 79, 8037-8045.

[20] Majors, P. D., McLean, J. S., Pinchuk, G. E., Fredrickson, J. K., et al., NMR methods for in situ biofilm metabolism studies. Journal of Microbiological Methods 2005, 62, 337-344. [21] Workentine, M., Harrison, J., Weljie, A., Tran, V., et al., Phenotypic and metabolic profiling of colony morphology variants evolved from Pseudomonas fluorescens biofilms. Environ Microbiol. 2010, 12, 1565-1577. 
[22] Spoering, A. L., Lewis, K., Biofilms and Planktonic Cells of Pseudomonas aeruginosa Have Similar Resistance to Killing by Antimicrobials. Journal of bacteriology 2001, 183, $6746-6751$.

[23] Pettit, R. K., Weber, C. A., Kean, M. J., Hoffmann, H., et al., Microplate Alamar blue assay for Staphylococcus epidermidis biofilm susceptibility testing. Antimicrob Agents Chemother 2005, 49, 2612-2617.

[24] Yu, F. P., McFeters, G. A., Physiological responses of bacteria in biofilms to disinfection. Appl Environ Microbiol 1994, 60, 2462-2466.

[25] von Stockar, U., Gustafsson, L., Larsson, C., Marison, I., et al., Thermodynamic Considerations In Constructing Energy Balances For Cellular Growth. Biochimica Et Biophysica Acta 1993, 1183, 221-240.

[26] von Stockar, U., Marison, I., The use of calorimetry in biotechnology, in Fiechter, A. (Ed.), Bioprocesses and Engineering, Springer Berlin / Heidelberg 1989, pp. 93-136.

[27] Maskow, T., Kemp, R., Buchholz, F., Schubert, T., et al., What heat is telling us about microbial conversions in nature and technology: from chip- to megacalorimetry. Microbial Biotechnology 2010, 3, 269-284.

[28] Peitzsch, M., Kiesel, B., Harms, H., Maskow, T., Real time analysis of Escherichia coli biofilms using calorimetry. Chem. Eng. Proc. 2007, 47, 1000-1006.

[29] von Rège, H., Sand, W., Evaluation of biocide efficacy by microcalorimetric determination of microbial activity in biofilms. Journal of Microbiological Methods 1998, 33, 227-235.

[30] Wentzien, S., Sand, W., Albertsen, A., Steudel, R., Thiosulfate and tetrathionate degradation as well as biofilm generation by Thiobacillus intermedius and Thiobacillus versutus studied by microcalorimetry, HPLC, and ion-pair chromatography. Archives of Microbiology 1994, 161, 116-125. 
[31] Weppen, P., Ebens, J., Müller, B. G., Schuller, D., On-line estimation of biological oxygen demand using direct calorimetry on surface attached microbial cultures.

Thermochimica Acta 1991, 193, 135-143.

[32] Barros, N., Feijo'o, S., A combined mass and energy balance to provide bioindicators of soil microbiological quality. Biophysical Chemistry 2003, 104, 561-572.

[33] Hauser-Gerspach, I., de Freitas, P. S., Dan Daniels, A. U., Meyer, J., Adhesion of Streptococcus sanguinis to glass surfaces measured by isothermal microcalorimetry (IMC). $J$ Biomed Mater Res B Appl Biomater 2008, 85, 42-49.

[34] Aulenta, F., Bassani, C., Ligthart, J., Majone, M., Tilche, A., Calorimetry: a tool for assessing microbial activity under aerobic and anoxic conditions. Water Research 2002, 36, 1297-1305.

[35] Senthilkumar, S., Surianarayanan, M., Swaminathan, G., Biocalorimetric and respirometric studies on biological treatment of tannery saline wastewater. Applied Microbiology and Biotechnology 2008, 78, 249-255.

[36] Lerchner, J., Wolf, A., Buchholz, F., Mertens, F., et al., Miniaturized calorimetry - a new method for real-time biofilm activity analysis. J Microbiol Methods 2008, 74, 74-81.

[37] Buchholz, F., Wolf, A., Lerchner, J., Mertens, F., et al., Chip calorimetry for fast and reliable evaluation of bactericidal and bacteriostatic treatments of biofilms. Antimicrob Agents Chemother 2010, 54, 312-319.

[38] Battley, E. H., Augustin Pierre Dubrunfaut. Thermochimica Acta 1998, 309, 1.

[39] Hill, A. V., A new form of differential microcalorimeter, for the estimation of heat production in physiological, bacteriological or ferment reactions. Jounal of Physiology 1911, $43,260-285$.

[40] Bayne-Jones, S., Bacterial Calorimetry I. General Considerations. Description of Differential Microcalorimeter. Journal of Bacteriology 1929, 17, 105-122.

[41] Gnaiger, E., Kemp, R. B., Anaerobic metabolism in aerobic mammalian cells: 
information from the ratio of calorimetric heat flux and respirometric oxygen flux. Biochim. Biophys. Acta 1990, 1016, 328-332.

[42] Bayne-Jones, S., Rhees, H. S., Bacterial Calorimetry II. Relationship of Heat Production to Phases of Growth of Bacteria. Journal of Bacteriology 1929, 17, 123-140.

[43] Birou, B., Marison, I. W., Stockar, U. V., Calorimetric investigation of aerobic fermentations. Biotechnol Bioeng 1987, 30, 650-660.

[44] Trampuz, A., Salzmann, S., Antheaume, J., Daniels, A. U., Microcalorimetry: a novel method for detection of microbial contamination in platelet products. Transfusion 2007, 47, 1643-1650.

[45] Trampuz, A., Steinhuber, A., Wittwer, M., Leib, S. L., Rapid diagnosis of experimental meningitis by bacterial heat production in cerebrospinal fluid. Bmc Infectious Diseases 2007, 7, 116-122.

[46] Baldoni, D., Hermann, H., Frei, R., Trampuz, A., Steinhuber, A., Performance of Microcalorimetry for Early Detection of Methicillin Resistance in Clinical Isolates of Staphylococcus aureus. J. Clin. Microbiol. 2009, 47, 774-776.

[47] Maskow, T., Harms, H., Real Time Insights into Bioprocesses Using Calorimetry: State of the Art and Potential. Engineering in Life Sciences 2006, 6, 266-277.

[48] Schill, N. A., Liu, J. S., Stockar, U., Thermodynamic analysis of growth of methanobacterium thermoautotrophicum. Biotechnol Bioeng 1999, 64, 74-81. [49] Schubert, T., Breuer, U., Harms, H., Maskow, T., Calorimetric bioprocess monitoring by small modifications to a standard bench-scale bioreactor. J Biotechnol 2007, 130, 24-31. [50] Postollec, F., Norde, W., van der Mei, H. C., Busscher, H. J., Microcalorimetric study on the influence of temperature on bacterial coaggregation. J Colloid Interface Sci 2005, 287, 461-467.

[51] Morgan, T. D., Beezer, A. E., An acrylic ampoule represents a denture surface in a microcalorimetric biofilm problem. Microbios 1998, 95, 55-64. 
[52] Sigstad, E. E., Bejas, M. A., Julia Amoroso, M., García, C. I., Effect of deforestation on soil microbial activity: A worm-composite can improve quality? A microcalorimetric analysis at $25{ }^{\circ} \mathrm{C}$. Thermochimica Acta 2002, 394, 171-178.

[53] Raich, J. W., Potter, C. S., Global Patterns of Carbon-Dioxide Emissions From Soils. Glob. Biogeochem. Cycle 1995, 9, 23-36.

[54] Barros, N., Salgado, J., Rodríguez-Añón, J., Proupín, J., et al., Calorimetric approach to metabolic carbon conversion efficiency in soils. Journal of Thermal Analysis and Calorimetry 2010, 99, 771-777.

[55] Kimura, T., Takahashi, K., Calorimetric Studies of Soil Microbes: Quantitative Relation between Heat Evolution during Microbial Degradation of Glucose and Changes in Microbial Activity in Soil. J Gen Microbiol 1985, 131, 3083-3089.

[56] Tissot, P., Calorimetric Study of the Bioremediation of a Polluted Soil. Journal of Thermal Analysis and Calorimetry 1999, 57, 303-312.

[57] Barros, N., Salgado, J., Feijóo, S., Calorimetry and soil. Thermochimica Acta 2007, 458, 11-17.

[58] Critter, S. A. M., Freitas, S. S., Airoldi, C., Calorimetry versus respirometry for the monitoring of microbial activity in a tropical soil. Applied Soil Ecology 2001, 18, 217-227. [59] Hansen, L. D., Macfarlane, C., McKinnon, N., Smith, B. N., Criddle, R. S., Use of calorespirometric ratios, heat per $\mathrm{CO} 2$ and heat per $\mathrm{O} 2$, to quantify metabolic paths and energetics of growing cells. Thermochimica Acta 2004, 422, 55-61.

[60] Buchholz, F., Wick, L. Y., Harms, H., Maskow, T., The kinetics of polycyclic aromatic hydrocarbon (PAH) biodegradation assessed by isothermal titration calorimetry (ITC).

Thermochimica Acta 2007, 458, 47-53.

[61] Mariana, F., Buchholz, F., Harms, H., Yong, Z., et al., Isothermal titration calorimetry -A new method for the quantification of microbial degradation of trace pollutants. Journal of Microbiological Methods 2010, 82, 42-48. 
[62] Johnston, M. D., Jones, M. V., Disinfection tests with intact biofilms: combined use of the Modified Robbins Device with impedance detection. Journal of Microbiological Methods $1995,21,15-26$.

[63] von Ah, U., Wirz, D., Daniels, A., Isothermal micro calorimetry--a new method for MIC determinations: results for 12 antibiotics and reference strains of E. coli and S. aureus. BMC Microbiol 2009, 9, 106-120.

[64] Baldoni, D., Steinhuber, A., Zimmerli, W., Trampuz, A., In vitro activity of gallium maltolate against Staphylococci in logarithmic, stationary, and biofilm growth phases: comparison of conventional and calorimetric susceptibility testing methods. Antimicrob Agents Chemother 2010, 54, 157-163.

[65] Mitik-Dineva, N., Wang, J., Mocanasu, R. C., Stoddart, P. R., et al., Impact of nanotopography on bacterial attachment. Biotechnology Journal 2008, 3, 536-544.

[66] Rosenhahn, A., Schilp, S., Kreuzer, H., Grunze, M., The role of "inert" surface chemistry in marine biofouling prevention. Phys Chem Chem Phys. 2010, 12, 4275-4286.

[67] Andersen, T. E., Kingshott, P., Palarasah, Y., Benter, M., et al., A flow chamber assay for quantitative evaluation of bacterial surface colonization used to investigate the influence of temperature and surface hydrophilicity on the biofilm forming capacity of uropathogenic Escherichia coli. Journal Of Microbiological Methods 2010, 81, 135-140.

[68] Cao-Hoang, L., Marechal, P. A., Lê-Thanh, M., Gervais, P., Waché, Y., Fluorescent probes to evaluate the physiological state and activity of microbial biocatalysts: A guide for prokaryotic and eukaryotic investigation. Biotechnology Journal 2008, 3, 890-903.

[69] Clauss, M., Trampuz, A., Borens, O., Bohner, M., Ilchmann, T., Biofilm formation on bone grafts and bone graft substitutes: Comparison of different materials by a standard in vitro test and microcalorimetry. Acta Biomater. 2010, 6, 3791-3797.

[70] Torres, F. E., Kuhn, P., De Bruyker, D., Bell, A. G., et al., Enthalpy arrays. Proceedings of the National Academy of Sciences of the United States of America 2004, 101, 9517-9522. 
[71] Chaires, J. B., Calorimetry and Thermodynamics in Drug Design. Annual Review of Biophysics 2008, 37, 135-151.

[72] Verhaegen, K., Baert, K., Simaels, J., Van Driessche, W., A high-throughput silicon microphysiometer. Sensors and Actuators A: Physical 2000, 82, 186-190.

[73] Mukhanov, V., Kemp, R., Design and experience of using light-emitting diodes (LEDs) as the inbuilt light source for a customised differential photomicrocalorimeter. Journal of Thermal Analysis and Calorimetry 2009, 95, 731-737. 


\section{Figure legends}

Fig.1: (A) TAM series; TA Instruments, USA) and (B) a chip-calorimeter

Fig.2: Heat output of T. intermedius growing on thiosulfate (reconstructed data from [30])

Fig. 3: Heat signal of an E. coli biofilm growing on glycerol. Rectangles indicate the ratio of TOC to the known carbon input (glycerol and acetate) in the outflow [28].

Fig.4: Heat responses of Pseudomonas putida biofilms towards the bacteriostatic antibiotic tetracycline and the bactericidal kanamyin and the biocide ( $2 \%$ formaldehyde) [37].

Fig. 5: Heat production rates from different soil samples, graphs reconstructed based on data from Barros et al. [32] 

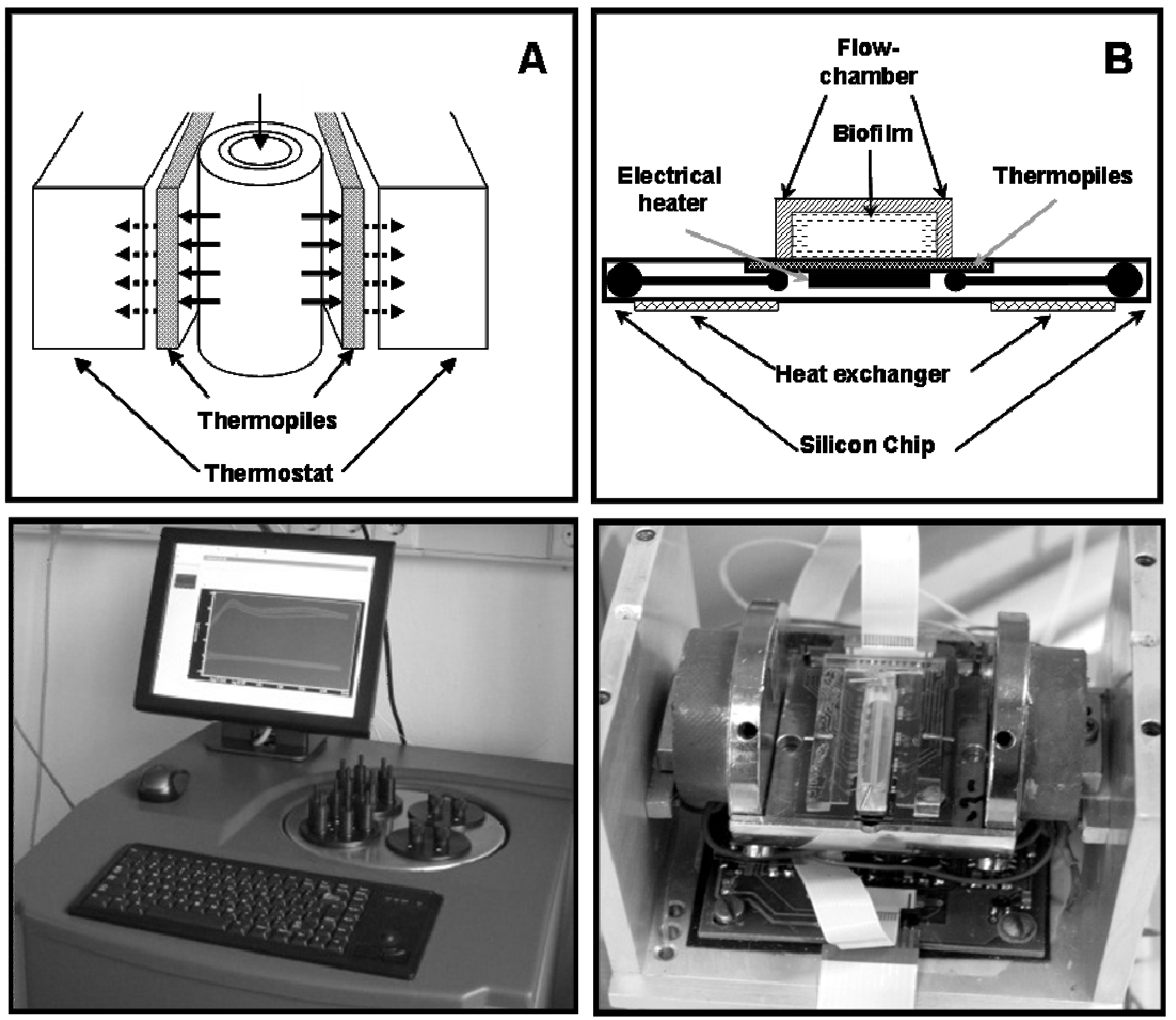

Wiley-VCH 
1

2

3

4

5

6

7

8

9

10

11

12

13

14

15

16

17

18

19

20

21

22

23

24

25

26

27

28

29

30

31

32

33

34

35

36

37

38

39

40

41

42

43

44

45

46

47

48

49

50

51

52

53

54

55

56

57

58

59

60

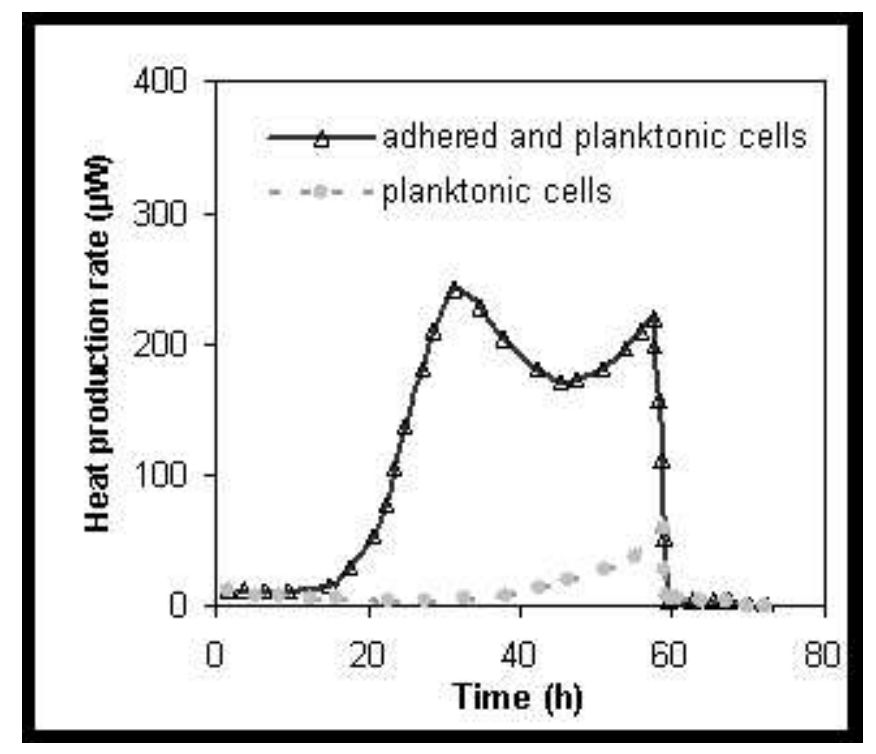

Heat output of $T$. intermedius growing on thiosulfate (reconstructed data from [30]) $85 \times 74 \mathrm{~mm}(96 \times 96$ DPI) 


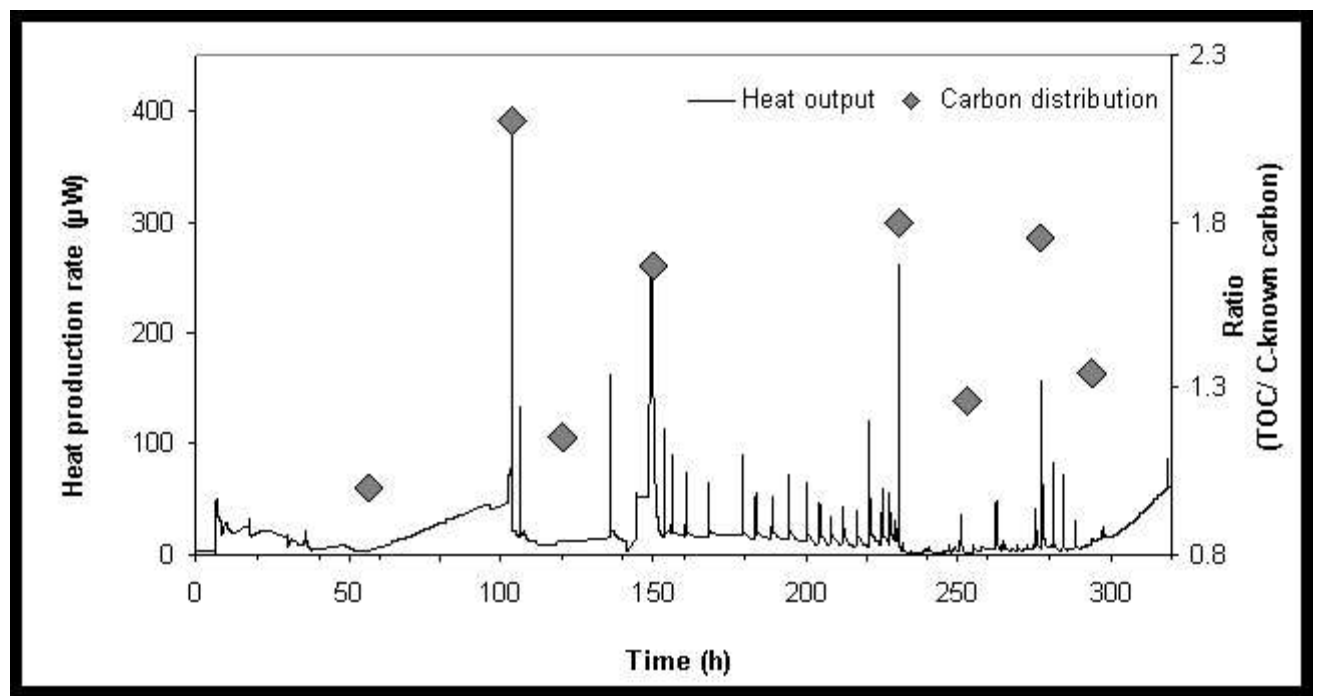

Heat signal of an E. coli biofilm growing on glycerol. Rectangles indicate the ratio of TOC to the known carbon input (glycerol and acetate) in the outflow [28]. $160 \times 84 \mathrm{~mm}(103 \times 103 \mathrm{DPI})$ 
1

2

3

4

5

6

7

8

9

10

11

12

13

14

15

16

17

18

19

20

21

22

23

24

25

26

27

28

29

30

31

32

33

34

35

36

37

38

39

40

41

42

43

44

45

46

47

48

49

50

51

52

53

54

55

56

57

58

59

60

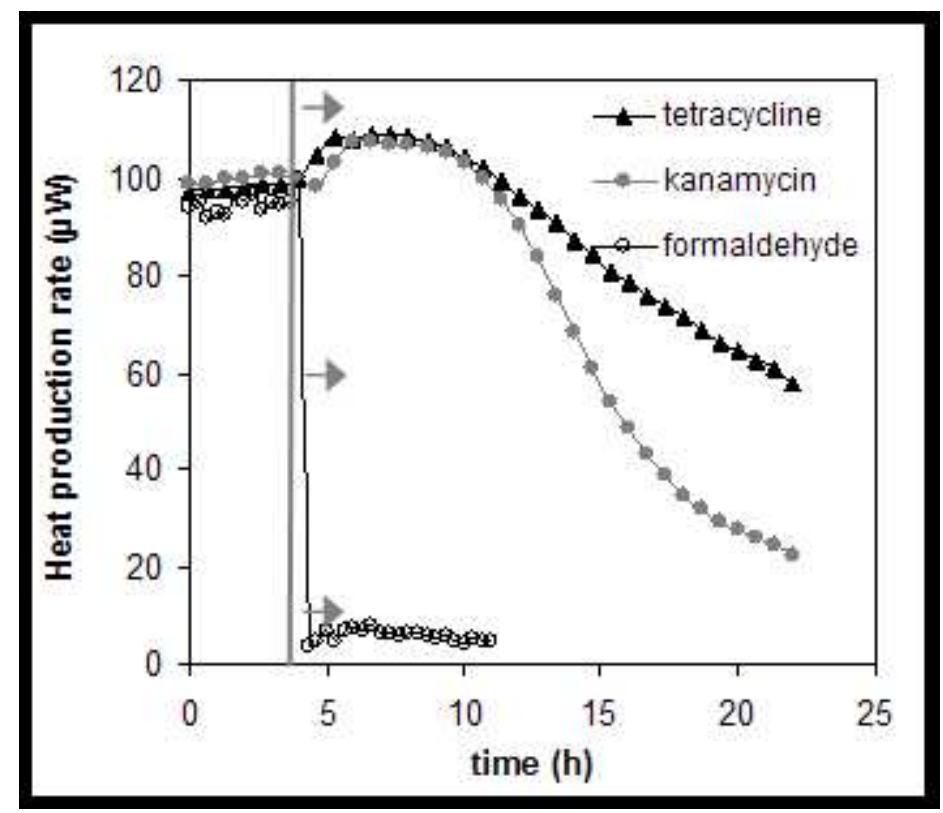

Heat responses of Pseudomonas putida biofilms towards the bacteriostatic antibiotic tetracycline and the bactericidal kanamyin and the biocide ( $2 \%$ formaldehyde) [37]. $93 \times 80 \mathrm{~mm}(96 \times 96 \mathrm{DPI})$ 
Heat production rates from different soil samples, graphs reconstructed based on data from Barros et al. [32]

$85 \times 74 \mathrm{~mm}(96 \times 96 \mathrm{DPI})$ 